\title{
The added value of intravoxel incoherent motion diffusion weighted imaging parameters in differentiating high-grade pancreatic neuroendocrine neoplasms from pancreatic ductal adenocarcinoma
}

\author{
WANLING MA $^{1 *}$, MENGQI WEI ${ }^{1 *}$, ZHIWEI HAN ${ }^{1}$, YONGQIANG TANG $^{1}$, \\ QI PAN ${ }^{2}$, GUANGWEN ZHANG ${ }^{1}$, JING REN ${ }^{1}$, YI HUAN ${ }^{1}$ and $\mathrm{NALI}^{3}$ \\ ${ }^{1}$ Department of Radiology, Xijing Hospital, Fourth Military Medical University, Xi'an, Shaanxi 710032; \\ ${ }^{2}$ Department of Radiology, Second Affiliated Hospital of Xi'an Medical Collage, Xi'an, Shaanxi 710038; \\ ${ }^{3}$ Department of Radiology, Ninth Hospital of Xi'an City, Xi'an, Shaanxi 710068, P.R. China
}

Received December 30, 2018; Accepted August 16, 2019

DOI: $10.3892 / 01.2019 .10863$

\begin{abstract}
The aim of the present study was to investigate the potential significance of intravoxel incoherent motion (IVIM)-diffusion weighted imaging (DWI) in differentiating high-grade pancreatic neuroendocrine neoplasms (pNENs) from pancreatic ductal adenocarcinoma (PDAC). A total of 50 patients, including 37 patients with PDAC and 13 patients with high-grade pNENs, underwent pancreatic multiple b-values DWI with 15 b-values including $0,10,20,40,60,80$, $100,150,200,400,800,1,000,1,200,1,500$ and $2,000 \mathrm{sec} / \mathrm{mm}^{2}$. Standard apparent diffusion coefficient $\left(\mathrm{ADC}_{\text {standard }}\right)$ and IVIM parameter [slow apparent diffusion coefficient $\left(D_{\text {slow }}\right)$, fast apparent diffusion coefficient $\left(\mathrm{D}_{\text {fast }}\right)$, fraction of fast apparent diffusion coefficient $(f)$ ] values of PDAC and pNENs were compared. $\mathrm{P}<0.05$ was considered to indicate a statistically
\end{abstract}

Correspondence to: Professor Na Li, Department of Radiology, Ninth Hospital of Xi'an City, 151 Second Ring South Road, Xi'an, Shaanxi 710068, P.R. China

E-mail: ln2002011039@163.com

${ }^{*}$ Contributed equally

Abbreviations: ADC, apparent diffusion coefficient; AUC, area under curve; DCE, dynamic contrast enhancement; DWI, diffusion weighted imaging; IVIM, intravoxel incoherent motion; IVIM-DWI, intravoxel incoherent motion diffusion weighted imaging; LAVA, liver acquisition with volume acceleration; pNENs, pancreatic neuroendocrine neoplasms; MVD, microvascular density; PDAC, pancreatic ductal adenocarcinoma; ROC, receiver operating characteristics; ROIs, regions of interest; SI, signal intensity

Key words: pancreatic ductal adenocarcinoma, pancreatic neuroendocrine neoplasms, intravoxel incoherent motion diffusion weighted imaging, slow apparent diffusion coefficient, fast apparent diffusion coefficient significant difference. Receiver operating characteristics analysis was performed in order to evaluate the diagnostic potential of IVIM parameters for differentiating high-grade pNENs from PDAC. $D_{\text {slow }}$ of pNENs was significantly lower compared with that of PDAC $\left(0.460\right.$ vs. $0.579 \times 10^{-3} \mathrm{~mm}^{2} / \mathrm{sec}$; $\mathrm{P}=0.001) . \mathrm{D}_{\text {fast }}$ of pNENs was significantly higher compared with that of PDAC (13.361 vs. $\left.4.985 \times 10^{-3} \mathrm{~mm}^{2} / \mathrm{sec} ; \mathrm{P}<0.001\right)$. Area under the curve of $\mathrm{D}_{\text {slow }}, \mathrm{D}_{\text {fast }}$ and combined $\mathrm{D}_{\text {slow }}$ and $\mathrm{D}_{\text {fast }}$ was $0.793,0.863$ and 0.885 respectively. The specificity and sensitivity of $D_{\text {slow }} \leq 0.472 \times 10^{-3} \mathrm{~mm}^{2} / \mathrm{sec}$ were 97.3 and $53.9 \%$, respectively, for differentiating high-grade pNENs from PDAC. The specificity and sensitivity of $\mathrm{D}_{\text {fast }}>9.58 \times 10^{-3} \mathrm{~mm}^{2} / \mathrm{sec}$ were 91.9 and $69.2 \%$, respectively, for differentiating high-grade pNENs from PDAC. When $D_{\text {slow }}$ and $D_{\text {fast }}$ were combined, the specificity and sensitivity for differentiating high-grade pNENs from PDAC were 76.9 and $100 \%$, respectively. Taken together, these results indicated that the diffusion-associated parameter $D_{\text {slow }}$ and the perfusion-associated parameter $D_{\text {fast }}$ of IVIM-DWI may differentiate high-grade pNENs from PDAC with high diagnostic accuracy, and that IVIM-DWI may be a valuable biomarker in differentiating pancreatic neoplasms.

\section{Introduction}

Pancreatic neuroendocrine neoplasms (pNENs) are the second most commonly-occurring solid neoplasms in the pancreas, accounting for $<3 \%$ of all pancreatic neoplasms (1). The reported annual incidence of pNENs is approximately 2.5-5 per million persons (2). As all pNENs have the potential to become malignant, surgery is the only curative treatment option for pNENs; thus, it is recommended to all patients (3-5). Compared with pancreatic ductal adenocarcinoma (PDAC), pNENs usually demonstrate more indolent biological behaviors; pNENs exhibit a more preferable response to chemotherapy, higher resectability and longer overall survival (6). Even advanced pNENs exhibit improved longer-term survival compared with PDAC (7). As PDAC is one of the leading causes of cancer-associated mortality worldwide, with mortality rates 
of $\sim 80.3 \%$ (8), preoperative adjuvant chemoradiation may improve the 1- and 2-year overall survival and disease free survival rates of patients (9). However, radiotherapy and chemotherapy induce numerous side effects, such as nausea, vomiting and bone marrow suppression. Therefore, it is imperative to accurately differentiate pNENs from PDAC prior to surgery.

Contrast-enhancement (CE) CT and MRI are widely accepted techniques for differentiating pNENs from PDAC. PNENs usually present with rapid and notable enhancement in the arterial phase of the CT scan due to their rich vascularity (7). However, pNENs gradually lose angiogenic potential and their microvascular density (MVD) decreases as the disease progresses (10). High-grade pNENs demonstrate lower vascularity and MVD compared with grade-1 pNENs $(11,12)$. Therefore, high-grade pNENs often exhibit hypo-enhancement or heterogeneous enhancement in early contrast-enhanced images (13). At present, the use of CE CT or MRI exhibit difficulties when differentiating high-grade pNENs from PDAC. Hypo-enhanced pNENs in arterial phase imaging are associated with poor differentiation, increased aggressiveness and decreased 5-year survival rate $(14,15)$. High-grade pNENs including grade 2 and 3 tumors often exhibit local invasion and/or metastases $(16,17)$.

Since Le Bihan et al (18) proposed the theory of intravoxel incoherent motion (IVIM), the phenomenon that tissue perfusion effects can be separated from true tissue diffusion in IVIM diffusion weighted imaging (IVIM-DWI) was determined $(19,20)$. Owing to the increasing concern for nephrogenic systemic fibrosis (21), IVIM-DWI may be an effective alternative to determine perfusion in tissues without contrast agents (22). An increasing number of studies have demonstrated the value of IVIM-DWI for evaluating pancreatic neoplasms $(23,24)$. In addition, studies have applied quantitative parameters derived from IVIM-DWI to predict histological characteristics of pNENs $(25,26)$. However, to the best of our knowledge, no published studies that compare IVIM-DWI parameters between pNENs and PDAC are currently available. Therefore, the aim of the present study was to assess the diagnostic performance of IVIM-DWI parameters for distinguishing between high-grade pNENs and PDAC.

\section{Materials and methods}

Subjects. Ethical approval was acquired from the Institutional Ethics Committee Board of Xijing hospital (Xi'an, China) and written informed consent was obtained from all participants. A total of 90 patients with suspected pancreatic solid mass were considered for inclusion in the present study between May 2014 and April 2017. A total of 40 patients were excluded due to the following exclusion criteria: i) Patients diagnosed with diseases other than PDAC and high-grade pNENs $(n=36)$ including grade 1 pNENs $(n=25)$, solid pseudopapillary tumor $(n=7)$, accessory spleen located in pancreas $(n=1)$, cholangiocarcinoma located in pancreatic segment of common bile duct $(n=2)$ and mass-forming chronic pancreatitis $(n=1)$; ii) patients lost to the follow-up $(n=4)$. The recruitment process is illustrated in Fig. 1.

Image acquisition. All patients were examined on a Discovery MR750 3.0 T whole-body MR scanner (GE Healthcare Life
Sciences) using a 32-channel phased-array coil. Pancreatic MR sequences comprised axial fast spin echo transverse relaxation time-weighted images $\left(\mathrm{T}_{2} \mathrm{WI}\right)$ with fat-suppression, axial breath-hold 3D liver acquisition with volume acceleration Flex (LAVA Flex) and LAVA Flex with contrast (Omniscan; GE Healthcare Life Sciences). Pancreatic multiple b-value diffusion-weighted echo-planar imaging was performed with 15 b-values, including 0, 10, 20, 40, 60, 80, 100, 150, 200, 400, $800,1,000,1,200,1,500$ and $2,000 \mathrm{sec} / \mathrm{mm}^{2}$. The detailed parameters of each sequence are presented in Table I.

Image and data analysis. An abdominal radiologist with 11 years of MRI experience blinded to the histopathological results analyzed the acquired IVIM-DWI data. Following examination, all data were transmitted to a built-in AW 4.6 workstation (GE Healthcare Life Sciences) for post-processing. Functional maps of IVIM parameters and a standard apparent diffusion coefficient $\left(\mathrm{ADC}_{\text {standard }}\right)$ map were processed by mean ADC (MADC) programs on the built-in AW 4.6 workstation.

$\mathrm{ADC}_{\text {standard }}$ was calculated using a mono-exponential model including the total $b$-values. The equation used was as follows: $\mathrm{S}(\mathrm{b}) / \mathrm{S}_{0}=\exp \left(-\mathrm{b} \times \mathrm{ADC}_{\text {standard }}\right.$ ) (equation 1). IVIM parameters including slow apparent diffusion coefficient $\left(D_{\text {slow }}\right)$, fast apparent diffusion coefficient $\left(D_{\text {fast }}\right)$ and fraction of fast apparent diffusion coefficient $(f)$ were calculated using bi-exponential fitting by the segmented fitting method. The equation used was as follows, as proposed by Le Bihan (20): $\mathrm{S}(\mathrm{b}) / \mathrm{S}_{0}=(1-f) \times \exp \left(-\mathrm{b} \times \mathrm{D}_{\text {slow }}\right)+f \times \exp \left(-\mathrm{b} \times \mathrm{D}_{\text {fast }}\right)$ (equation 2), where $S(b)$ represents the mean signal intensity (SI) of a DW image according to a specific b-value, $S_{0}$ represents the mean SI of a DW image when $b=0, D_{\text {slow }}$ represents true tissue diffusivity while tissue microcapillary perfusion is mainly excluded, $\mathrm{D}_{\text {fast }}$ represents the mean velocity of the flowing blood within capillaries and the microvascular architecture and $f$ represents the ratio of molecular diffusion within capillaries compared with the overall water molecular diffusion in a voxel. As $\mathrm{D}_{\text {fast }}$ is larger compared with $\mathrm{D}_{\text {slow }}$ by approximately one order of magnitude (22), - b x $\mathrm{D}_{\text {fast }}$ would be $>-3$ when the $b>200 \mathrm{sec} / \mathrm{mm}^{2}$, and the $f \times \exp \left(-b \times D_{\text {fast }}\right)$ value would be $>0.05 \times f$. Therefore, $f \times \exp \left(-\mathrm{b} \times \mathrm{D}_{\text {fast }}\right)$ can be neglected and equation 2 can be expressed as follows: $\mathrm{S}(\mathrm{b}) / \mathrm{S}_{0}=(1-f) \times \exp \left(-\mathrm{b} \times \mathrm{D}_{\text {slow }}\right)$ (equation 3). As b-values were $>200 \mathrm{sec} / \mathrm{mm}^{2}, \mathrm{~S}(\mathrm{~b})$ was fitted using equation 3 according to a linear model, and $\mathrm{D}_{\text {slow }}$ was calculated. The $f$ value was previously calculated according to equation 3 ; however, the accuracy was unacceptable. Therefore, the $f$-value was recalculated according to equation 2 . Subsequently, $\mathrm{S}(\mathrm{b})$ was fitted for all $b$-values according to equation 2 with the $D_{\text {slow }}$ value fixed by the nonlinear Levenberg-Marquardt method (19). When equation 2 was fitted, the initial estimated $D_{\text {fast }}$ value was set as $10 \times 10^{-3} \mathrm{~mm}^{2} / \mathrm{sec}$, and the $f$-value was set as the previous $f$-value calculated from equation 3 . The $\mathrm{D}_{\text {fast }}$ and $f$ values were then acquired.

On the DW images that distinctly displayed the tumors, irregular regions of interest (ROIs) for each PDAC and pNENs were manually delineated along the edge of the tumor on three consecutive largest lesion slices. During measurement, particular efforts were made to exclude any areas of necrosis, the pancreatic duct and vessels within the tumor. Well-matched ROI copies were generated synchronously and appeared 


\section{0 patients with a suspect focal pancreatic solid mass seen in CT examination}

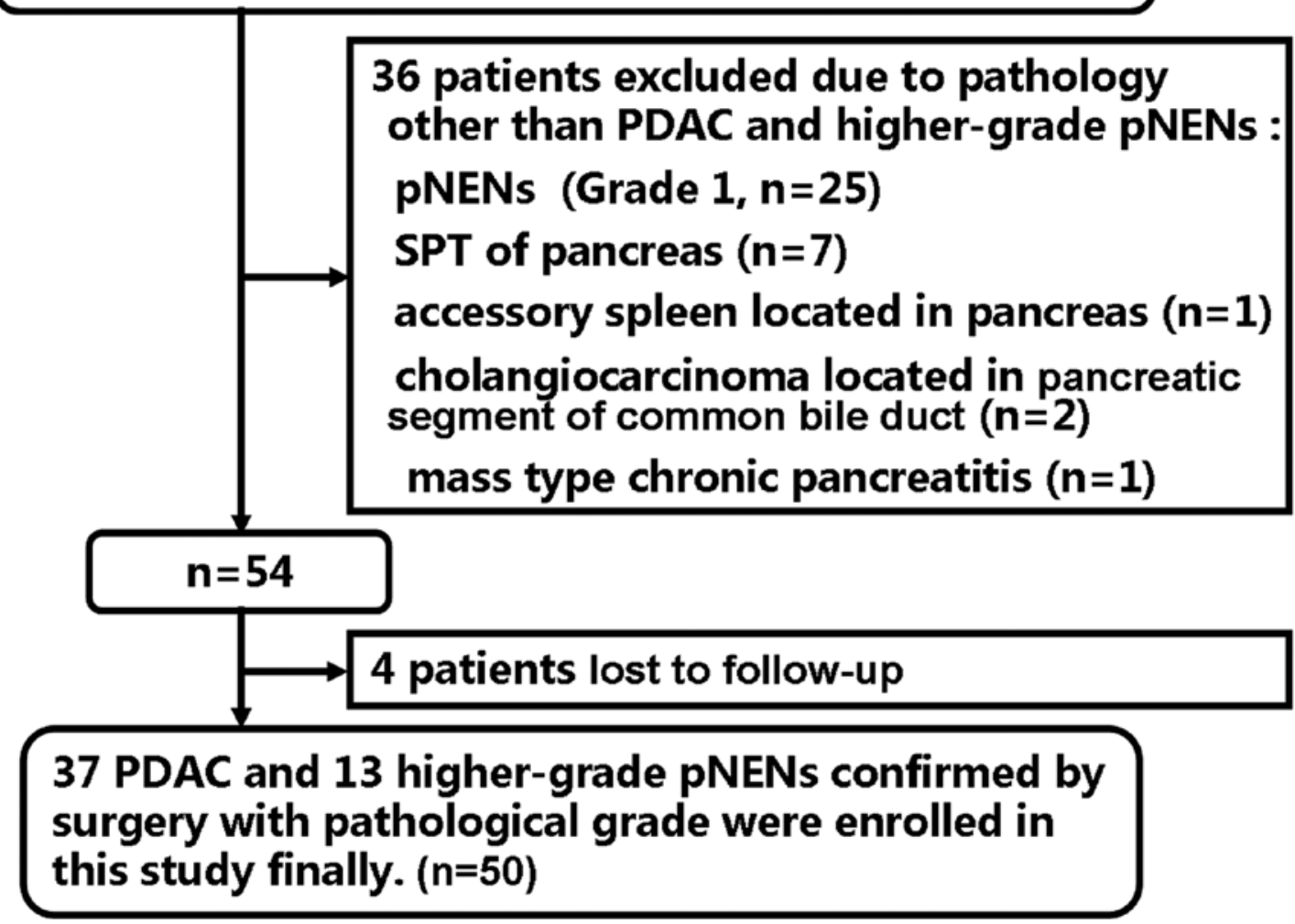

Figure 1. Flowchart of the study enrollment process. Of the 90 patients, 50 patients with histopathologically confirmed PDAC and pNENs were enrolled. PDAC, pancreatic ductal adenocarcinoma; pNENs, pancreatic neuroendocrine neoplasms; SPT, solid pseudopapillary tumor.

automatically on identical locations in every functional map of IVIM-DWI parameters and $\mathrm{ADC}_{\text {standard }}$ by built-in MADC software on an AW 4.6 workstation (GE Healthcare Life Sciences). The processes of ROI setting on functional parameter maps are presented in Fig. 2. The mean value of the results of three measurements was used as the final result. The ROI area range of pNENs was between 22 and $1,379 \mathrm{~mm}^{2}$ with a mean area of $372.22 \mathrm{~mm}^{2}$. The ROI area range of PDAC was between 136 and $1,025 \mathrm{~mm}^{2}$ with a mean area of $324.58 \mathrm{~mm}^{2}$.

Statistical analysis. All analyses were performed using SPSS software version 17.0 for Windows (SPSS Inc.) and MedCalc software version 12.3 (MedCalc Software). The $\mathrm{ADC}_{\text {standard }}$ and IVIM-DWI parameter values are presented as the mean \pm standard deviation. $\mathrm{ADC}_{\text {standard }}$ and IVIM-DWI parameter values were compared using an independent sample Student's t-test. Receiver operating characteristics (ROC) analysis was performed to evaluate the diagnostic performance of IVIM-DWI parameters and to determine the cut-off values using the maximum Youden index (the sum of specificity and sensitivity). $\mathrm{P}<0.05$ was considered to indicate a statistically significant difference.

\section{Results}

Clinical and pathological characteristics of patients and lesions. The clinical and pathological characteristics of patients and lesions are summarized in Table II. A total of 50 patients including 13 cases of high-grade pNENs (grade 2, $\mathrm{n}=11$; grade $3, \mathrm{n}=2$ ) and 37 cases of PDAC (well/moderately differentiated, $n=21$; poorly differentiated, $n=11$; confirmed by biopsy without pathological grades, $n=5$ ) confirmed by surgical pathology were included (27). The pNENs cohort (mean age, 55.1 years; age range, $42-75$ years) included 8 male and 5 female patients. The clinical symptoms of the pNENs group included hypoglycemia, epigastric pain and discomfort, thrombocytopenia and splenomegaly. Among the pNENs group, one patient presented with painless jaundice. The PDAC cohort (mean age, 57.5 years; age range, 20-76 years) included 23 male and 14 female patients. The clinical symptoms of the PDAC group included marasmus, dyspepsia and abdominal and back pain. Among the PDAC group, 11 patients presented with jaundice.

Conventional MRI results. The conventional MRI results of PDAC and pNENs are presented in Table III. The mean long diameter of pNENs was $3.31 \mathrm{~cm}$ (range, 0.89-5.58 cm). Among the pNENs, 7 lesions were located in the head, 2 were in the body and 4 were in the tail of the pancreas. In the $\mathrm{T}_{2} \mathrm{~W}$ images, 7 pNENs exhibited moderate hyperintensity, 4 exhibited slight hyperintensity and 2 exhibited moderate hypointensity. In the LAVA Flex water phase images, 12 pNENs exhibited moderate hypointensity and 1 presented with slight hypointensity. In the LAVA Flex with contrast 
Table I. MRI parameters.

\begin{tabular}{lccc}
\hline & & Sequence & \\
\cline { 2 - 4 } Parameters & Axial FSE T,WI & Axial LAVA Flex & 6600 \\
\hline Repetition time, ms & 10,000 & 4.3 & $81.5-82.3$ \\
Echo time, ms & 70 & 1.6 & 4.0 \\
Slice thickness, mm & 4.0 & 4.0 & 1.0 \\
Slice gap, mm & 0.5 & 0 & $128 \times 128 \times$ NS \\
Matrix size, slices & $320 \times 320 \times$ NS & $360 \times 324$ & $380 \times 304$ \\
Field of view, mm & $360 \times 360$ & 1 & $1-8$ \\
Number of excitations ${ }^{\mathrm{a}}$ & 1.5 & 14 & 90 \\
Flip angle, ${ }^{\circ}$ & 110 & 200 & 250 \\
Bandwidth, Hz/pixel & 62.5 & 11 & $480-660$ \\
Acquisition Time, sec & $120-240$ & & \\
\hline
\end{tabular}

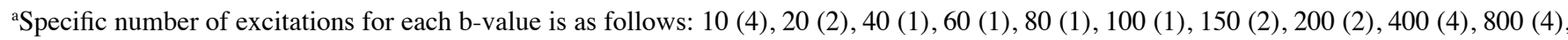
$1,000(6), 1,200(6), 1,500(6)$ and 2,000 (8) $\mathrm{s} / \mathrm{mm}^{2}$; the numbers in brackets represent the number of excitations. FSE, fast spin echo; $\mathrm{T}_{2} \mathrm{WI}$, fast spin echo transverse relaxation time-weighted imaging; LAVA, liver acquisition with volume acceleration; DWI, diffusion weighted imaging; NS, number of slices.

images, all pNENs exhibited heterogeneous mild-to-moderate hypo-enhancement on arterial phase imaging. Representative conventional images of pNENs are presented in Fig. 3A-C. In the DW images, 12 pNENs exhibited hyperintensity and 1 displayed hypointensity. Among the 12 hyperintensive pNENs, 5 (41.7\%) exhibited heterogeneous SI and 7 (58.3\%) exhibited homogeneous SI in DWI. In addition, 8 of 12 $(66.7 \%)$ hyperintensive pNENs exhibited high SI in all b-value DW images, including low b-value ( $\left.b \leq 200 \mathrm{sec} / \mathrm{mm}^{2}\right)$, moderate $b$-value $\left(200 \mathrm{sec} / \mathrm{mm}^{2}<\mathrm{b} \leq 1,500 \mathrm{sec} / \mathrm{mm}^{2}\right)$ and high b-value $\left(b>1,500 \mathrm{sec} / \mathrm{mm}^{2}\right)$. In addition, 4 of $12(33.3 \%)$ hyperintensive pNENs demonstrated high SI in low and moderate b-value DW images; however, they exhibited low SI in high b-value DW images. Representative DW images of pNENs are presented in Fig. 3D-F.

The mean long diameter of PDAC was $4.03 \mathrm{~cm}$ (range, 2.05-6.82 cm). Among the PDAC cohort, 20 lesions were located in the head, 6 in the neck, 8 in the body and 3 in the tail of the pancreas. In the $\mathrm{T}_{2} \mathrm{~W}$ images, 29 PDAC exhibited moderate hyperintensity, 5 exhibited slight hyperintensity, 1 exhibited isointensity and 2 exhibited slight hypointensity. In the LAVA Flex water phase images, 26 PDAC exhibited moderate hypointensity, 7 exhibited slight hypointensity, 1 exhibited slight hyperintensity and 3 exhibited isointensity. In the LAVA Flex with contrast images, all PDAC exhibited heterogeneous hypo-enhancement with different degrees in arterial phase imaging. Representative conventional images of PDAC are presented in Fig. 4A-C. In the DW images, 36 PDAC cases exhibited significant hyperintensity and 1 exhibited slight hypointensity. In addition, 27 of 36 (75\%) hyperintensive PDAC exhibited heterogeneous SI and $9(25 \%)$ indicated homogeneous SI in DWI. Among the 36 hyperintensive PDAC, 27 (75\%) demonstrated high SI in all b-value DW images; however, $9(25 \%)$ exhibited high SI in low and moderate b-value DW images only. Representative DW images of PDAC are presented in Fig. 4D-F.
Comparison of IVIM-DWI parameters. IVIM-DWI parameters of PDAC and pNENs are presented in Table IV. The mean $\mathrm{D}_{\text {slow }}$ value was significantly lower in pNENs compared with that in PDAC $\left(0.460 \times 10^{-3}\right.$ vs. $0.579 \times 10^{-3} \mathrm{~mm}^{2} / \mathrm{sec}$; $\mathrm{t}=3.509$; $\mathrm{P}=0.001$ ) (Fig. $5 \mathrm{~A}$ ), whereas the mean $\mathrm{D}_{\text {fast }}$ value was significantly higher in pNENs compared with that in PDAC (13.361 $\times 10^{-3}$ vs. $\left.4.985 \times 10^{-3} \mathrm{~mm}^{2} / \mathrm{sec} ; \mathrm{t}=5.071 ; \mathrm{P}<0.001\right)$ (Fig. 5B). For $\mathrm{ADC}_{\text {standard }}$ and $f$, no significant differences were observed between pNENs and PDAC $\left(0.818 \times 10^{-3}\right.$ vs. $0.863 \times 10^{-3} \mathrm{~mm}^{2} / \mathrm{sec} ; \mathrm{t}=0.961 ; \mathrm{P}=0.341 ;$ and 59.5 vs. $55.8 \%$; $\mathrm{t}=0.872 ; \mathrm{P}=0.388$, respectively) (Fig. $5 \mathrm{C}$ and $\mathrm{D}$ ).

ROC curves for differentiating pNENs from PDAC using $D_{\text {slow }}$ and $D_{\text {fast }}$ are presented in Fig. 6. The optimal cut-off values and AUC in differentiating pNENs from PDAC are listed in Table V. The AUC for $\mathrm{D}_{\text {fast }}$ was slightly larger compared with that for $\mathrm{D}_{\text {slow }}(0.863$ vs. $0.793 ; \mathrm{P}=0.499)$, whereas the AUC for combined $D_{\text {slow }}$ and $D_{\text {fast }}$ was slightly larger compared with that for $\mathrm{D}_{\text {slow }}$ or $\mathrm{D}_{\text {fast }}$ alone (0.885 vs. $0.793 ; \mathrm{P}=0.257$; 0.885 vs. $0.863 ; \mathrm{P}=0.757$, respectively). When $\mathrm{D}_{\text {slow }}$ value was $\leq 0.472 \times 10^{-3} \mathrm{~mm}^{2} / \mathrm{sec}$, the specificity and sensitivity for differentiating pNENs from PDAC were 97.3 and $53.9 \%$, respectively. When $D_{\text {fast }}$ value was $>9.58 \times 10^{-3} \mathrm{~mm}^{2} / \mathrm{sec}$, the specificity and sensitivity for differentiating pNENs from PDAC were 91.9 and $69.2 \%$, respectively. When $\mathrm{D}_{\text {slow }}$ and $\mathrm{D}_{\text {fast }}$ were combined, the specificity and sensitivity for differentiating high-grade pNENs from PDAC were 76.9 and $100 \%$, respectively.

\section{Discussion}

The present study identified statistically significant differences in $\mathrm{D}_{\text {fast }}$ and $\mathrm{D}_{\text {slow }}$ between PDAC and pNENs. The differences in $\mathrm{ADC}_{\text {standard }}$ and $f$ exhibited no significance between PDAC and pNENs. The ADC value is the most commonly used parameter for evaluating tissue diffusion that serves as a marker of cellularity $(28,29)$, which is additionally decreased 

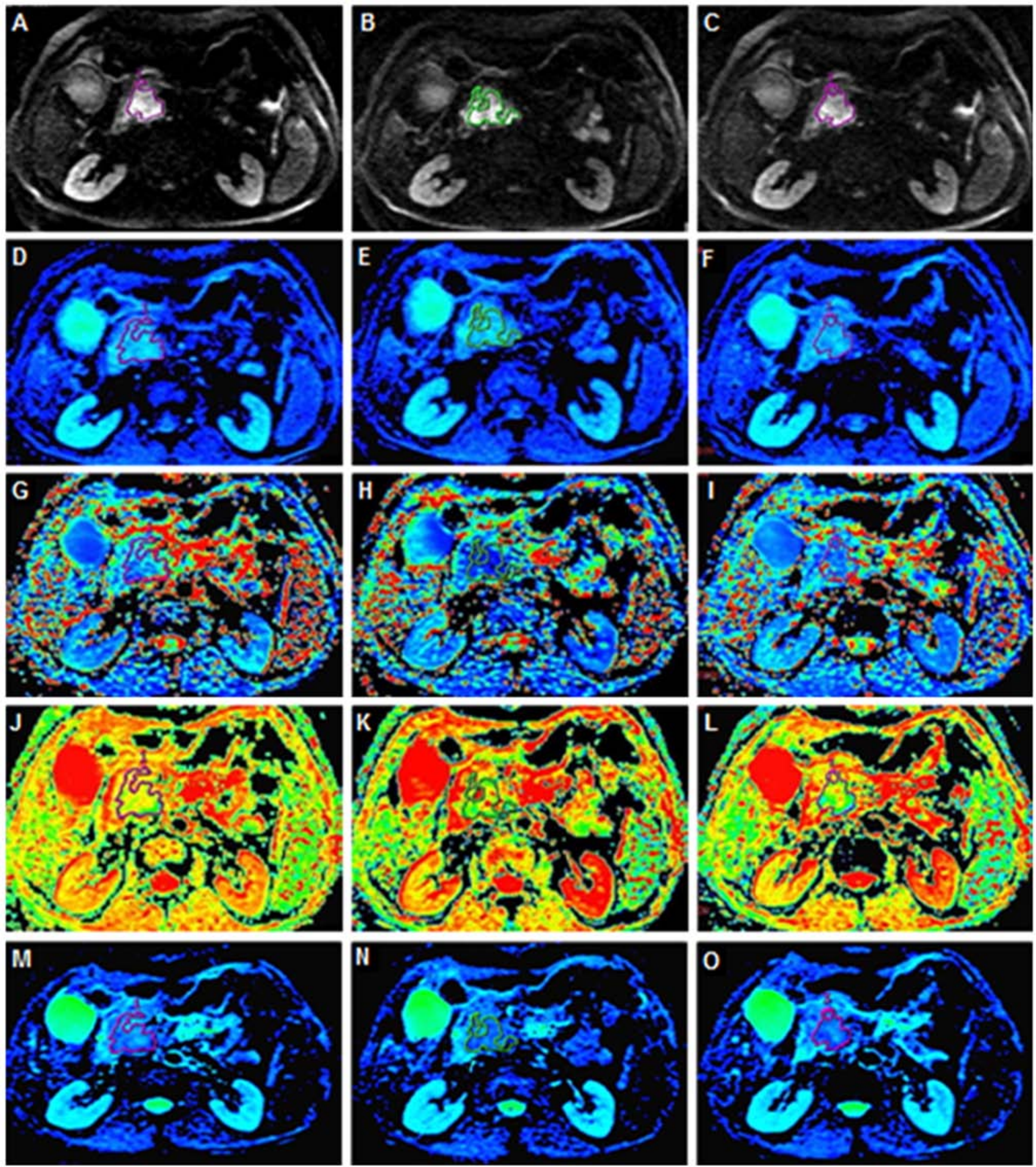

Figure 2. Different functional parameter maps and ROI setting. Irregular ROIs represent the tumor area on three of the largest consecutive lesion slices. (A-C) Axial DWI with a b-value of $1,200 \mathrm{~s} / \mathrm{mm}^{2}$. (D-F) Functional parameter pseudo-color images of $\mathrm{D}_{\text {slow. }}$ (G-I) Functional parameter pseudo-color images of $\mathrm{D}_{\text {fast }}$. (J-L) Functional parameter pseudo-color images of $f$. (M-O) Functional parameter pseudo-color images of ADC standard ROI, region of interest; DWI, diffusion weighted imaging; ADC, apparent diffusion coefficient; $\mathrm{D}_{\text {slow }}$, slow apparent diffusion coefficient; $\mathrm{D}_{\text {fast }}$, fast apparent diffusion coefficient; $f$, fraction of fast apparent diffusion coefficient.

in solid malignant tumors compared with benign tumors or cystic lesions (30). The ADC value can be a surrogate biomarker for tumor cell proliferation and predict the grade of a variety of neoplasms $(31,32)$. However, previous studies have demonstrated that not both water diffusion within tissue microstructures and the undirected movement of particles within the capillaries can influence the obtained ADC value $(19,33)$. The present study revealed that the $\mathrm{ADC}_{\text {standard }}$ of PDAC was slightly higher compared with that of pNENs. The measurement of the ADC value is biased by the effects of microcirculatory perfusion, which may impact the accuracy of ADC in evaluating pancreatic lesions (33).

The IVIM-derived parameter $D_{\text {slow }}$ represents the pure diffusion component reflecting tissue microstructure without 
Table II. Clinicopathological characteristics of patients and lesions.

\begin{tabular}{lcc}
\hline Characteristics & PDAC & pNENs \\
\hline Sex, $\mathrm{n}$ & 23 & 8 \\
Male & 14 & 5 \\
Female & $\mathrm{a}$ & $\mathrm{b}$ \\
Grading & Well/moderately & Grade 2 \\
& differentiated (21) & $(11)$ \\
& Poorly & Grade 3 \\
& differentiated (11) & $(2)$ \\
& Confirmed by biopsy & \\
& without pathological & \\
Clinical symptoms & grades (5) & 1 \\
Painless jaundice & & 3 \\
Hypoglycemia & 11 & 6 \\
Epigastric pain & 0 & 1 \\
and discomfort & 0 & 0 \\
Thrombocytopenia & & \\
and splenomegaly & 0 & \\
Marasmus & & \\
Dyspepsia & & \\
Abdominal and & & \\
back pain & & \\
\hline
\end{tabular}

PDAC, pancreatic ductal adenocarcinoma; pNENs, pancreatic neuroendocrine neoplasms. ${ }^{a}$ According to 2010 WHO Classification of Tumors of Digestive System (27). ${ }^{\text {b} A c c o r d i n g ~ t o ~} 2017$ World Health Organization Neuroendocrine Tumor Classification Guideline (3).

perfusion effects (34). The predominant histological features of PDAC include not only the dense tumor cellularity, but also progressive fibrosis (35). Restrained molecular diffusion in PDAC may be attributed to an exceeding cellular structure and extracellular fibrosis (36). Previous studies have demonstrated that the lower $\mathrm{D}_{\text {slow }}$ value of pNENs (grade 2 or 3 ) may be caused by the increased tumor cellularity $(25,37)$. However, necrosis is also one of the pathological features in PDAC and may be associated with increasing diffusion (30). The results of the present study demonstrated that $75.0 \%$ hyperintensive PDAC appeared as heterogeneous SI in DW images, whereas only $41.7 \%$ hyperintensive pNENs exhibited heterogeneous SI in DW images. This may be the cause for the results obtained in the present study, as the $\mathrm{D}_{\text {slow }}$ of PDAC was significantly higher compared with that of $\mathrm{pNENs}$. Therefore, $\mathrm{D}_{\text {slow }}$ may be more useful compared with conventional ADC in differentiating pNENs and PDAC by eliminating microperfusion effects in a capillary bed.

The results of the present study demonstrated that $\mathrm{D}_{\text {fast }}$ of PDAC was notably lower compared with that of pNENs. The $f$ of PDAC was slightly lower compared with that of pNENs. $D_{\text {fast }}$ is associated with blood flow and velocity within microcirculation $(19,22)$. Parameter $f$ is associated with the proportion of protons in microcirculation within a voxel (29).
Table III. Conventional magnetic resonance imaging results.

\begin{tabular}{|c|c|c|}
\hline Characteristic & PDAC, $\mathrm{n}$ & pNENs, $n$ \\
\hline \multicolumn{3}{|l|}{ Site } \\
\hline Head of pancreas & 20 & 7 \\
\hline Neck of pancreas & 6 & 0 \\
\hline Body of pancreas & 8 & 2 \\
\hline Tail of pancreas & 3 & 4 \\
\hline \multicolumn{3}{|l|}{$\mathrm{T}_{2} \mathrm{~W}$ images } \\
\hline Moderate hyperintensity & 29 & 7 \\
\hline Slight hyperintensity & 5 & 4 \\
\hline Isointensity & 1 & 0 \\
\hline Moderate hypointensity & 0 & 2 \\
\hline Slight hypointensity & 2 & 0 \\
\hline \multicolumn{3}{|l|}{ LAVA Flex water phase images } \\
\hline Moderate hypointensity & 26 & 12 \\
\hline Slight hypointensity & 7 & 1 \\
\hline Isointensity & 3 & 0 \\
\hline Slight hyperintensity & 1 & 0 \\
\hline \multicolumn{3}{|l|}{ LAVA Flex with contrast images } \\
\hline $\begin{array}{l}\text { Heterogeneous hypo-enhancement } \\
\text { with a different degree }\end{array}$ & 37 & 13 \\
\hline \multicolumn{3}{|l|}{ DWI } \\
\hline Hyperintensity & 36 & 12 \\
\hline All b-values & 27 & 8 \\
\hline $\begin{array}{l}\text { Low and moderate b-value } \\
\left(0<\mathrm{b} \leq 1,500 \mathrm{sec} / \mathrm{mm}^{2}\right)\end{array}$ & 9 & 4 \\
\hline Hypointensity & 1 & 1 \\
\hline
\end{tabular}

PDAC, pancreatic ductal adenocarcinoma; pNENs, pancreatic neuroendocrine neoplasms; LAVA, liver acquisition with volume acceleration; DWI, diffusion weighted imaging; $\mathrm{T}_{2} \mathrm{~W}$, fast spin echo transverse relaxation time-weighted.

Poor blood supply leads to a lower blood volume and flow in PDAC compared with a normal pancreas (38). Due to abundant fibrosis and relatively sparse vascularity of PDAC, the enhancement degree of PDAC is lower compared with that of normal pancreatic tissue, especially in early post-contrast images $(35,38)$. However, pNENs typically demonstrate fast and strong enhancement in early contrast-enhanced images due to abundant vascularity (7,39). A study by Kang et al (24) demonstrated that tissue perfusion may be a more important factor compared with diffusion in differentiating pNENs from PDAC. The results of the present study indicated that $D_{\text {fast }}$ and $f$ of pNENs were higher compared with those of PDAC due to different vascular perfusion, which was in agreement with the results obtained by Kang et al (24), although no significant differences in $f$ were identified between pNENs and PDAC. High-grade pNENs including grade 2 and 3 exhibit a lower vascularity and MVD (11-12), which leads to a decreased $f$ value of pNENs, similar to that of PDAC. Unlike the study by Kang et al (24), which stated that $\mathrm{D}_{\text {slow }}$ was not useful in differentiating PDAC and pNENs, the results obtained in the present study demonstrated that the $\mathrm{D}_{\text {slow }}$ of PDAC was 

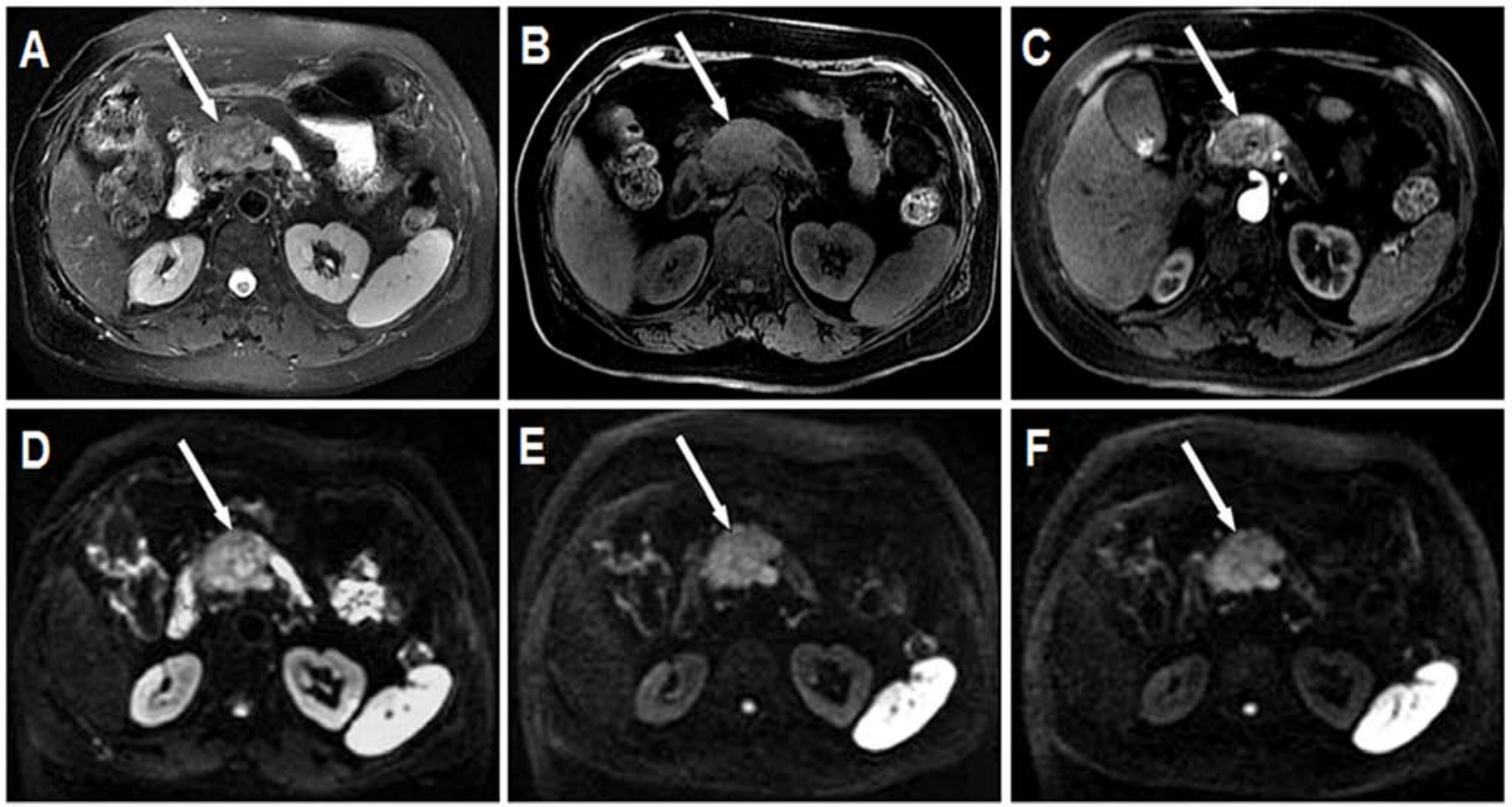

Figure 3. Grade $2 \mathrm{pNEN}$ located in the head of the pancreas (white arrow). The pancreatic body and tail exhibit atrophy and the pancreatic duct exhibits dilation. (A) Axial $\mathrm{T}_{2} \mathrm{~W}$ MRI with fat suppression demonstrates pNEN with heterogeneous moderate hyperintensity and a relatively clear boundary. (B) Axial LAVA Flex water phase image displays pNEN with heterogeneous moderate hypointensity and an obscure boundary. (C) Axial arterial phase image demonstrates pNEN with mild heterogeneous enhancement. (D) Axial DWI with a b-value of $200 \mathrm{~s} / \mathrm{mm}^{2}$ demonstrates pNEN with heterogeneous hyperintensity and an obscure boundary. (E) Axial DWI with a b-value of 1,500s/ $\mathrm{mm}^{2}$ demonstrates pNEN with heterogeneous hyperintensity and a clear boundary. (F) Axial DWI with a b-value of $2,000 \mathrm{~s} / \mathrm{mm}^{2}$ demonstrates pNEN with heterogeneous hyperintensity and a clear boundary. pNEN, pancreatic neuroendocrine neoplasm; LAVA, liver acquisition with volume acceleration; DWI, diffusion weighted imaging; $\mathrm{T}_{2} \mathrm{~W}$, fast spin echo transverse relaxation time-weighted.
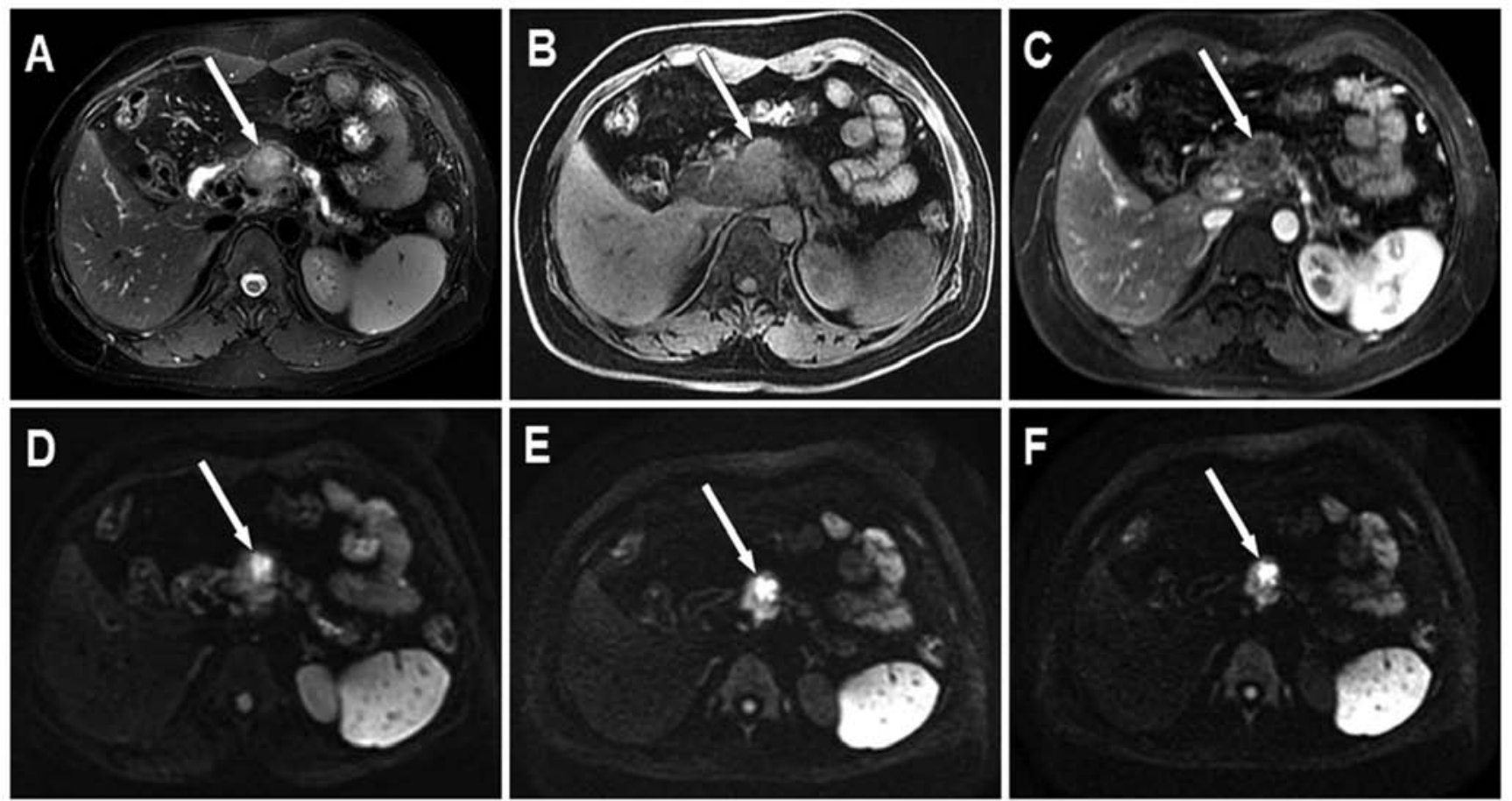

Figure 4. PDAC located in the head of the pancreas (white arrow). The pancreatic body and tail exhibit atrophy and the pancreatic duct presents with dilation. (A) Axial $\mathrm{T}_{2} \mathrm{~W}$ MRI with fat suppression displays PDAC with heterogeneous moderate hyperintensity and an obscure boundary. (B) Axial LAVA Flex water phase image demonstrates PDAC with heterogeneous moderate hypointensity and an obscure boundary. (C) Axial arterial phase image demonstrates PDAC with mild heterogeneous enhancement. (D) Axial DWI with b-value of 200s $/ \mathrm{mm}^{2}$ demonstrates PDAC with heterogeneous hyperintensity and a clear boundary. (E) Axial DWI with a b-value of $1,500 \mathrm{~s} / \mathrm{mm}^{2}$ demonstrates PDAC with heterogeneous hyperintensity and a clear boundary. (F) Axial DWI with a b-value of $2,000 \mathrm{~s} / \mathrm{mm}^{2}$ demonstrates PDAC with heterogeneous hyperintensity and a clear boundary. DWI, diffusion weighted imaging; PDAC, pancreatic ductal adenocarcinoma; LAVA, liver acquisition with volume acceleration; $\mathrm{T}_{2} \mathrm{~W}$, fast spin echo transverse relaxation time-weighted. 
Table IV. Intravoxel incoherent motion and diffusion weighted imaging parameters of PDAC and pNENs.

\begin{tabular}{|c|c|c|c|c|}
\hline Coefficient & $\operatorname{PDAC}(\mathrm{n}=37)$ & pNENs $(n=13)$ & t-value & $\mathrm{P}$-value \\
\hline $\mathrm{ADC}_{\text {standard }}\left(\mathrm{x} 10^{-3} \mathrm{~mm}^{2} / \mathrm{sec}\right)$ & $0.863 \pm 0.152$ & $0.818 \pm 0.120$ & 0.961 & 0.341 \\
\hline $\mathrm{D}_{\text {slow }}\left(\mathrm{x} 10^{-3} \mathrm{~mm}^{2} / \mathrm{sec}\right)$ & $0.579 \pm 0.104$ & $0.460 \pm 0.108$ & 3.509 & 0.001 \\
\hline $\mathrm{D}_{\text {fast }}\left(\times 10^{-3} \mathrm{~mm}^{2} / \mathrm{sec}\right)$ & $4.985 \pm 3.589$ & $13.361 \pm 8.145$ & 5.071 & $<0.001$ \\
\hline$f(\%)$ & $55.8 \pm 13.6$ & $59.5 \pm 10.1$ & 0.872 & 0.388 \\
\hline
\end{tabular}

PDAC, pancreatic ductal adenocarcinoma; pNENs, pancreatic neuroendocrine neoplasms; $\mathrm{ADC}_{\text {standard }}$, standard apparent diffusion coefficient; $\mathrm{D}_{\text {slow }}$, slow apparent diffusion coefficient; $\mathrm{D}_{\text {fast }}$, fast apparent diffusion coefficient; $f$, fraction of fast apparent diffusion coefficient.
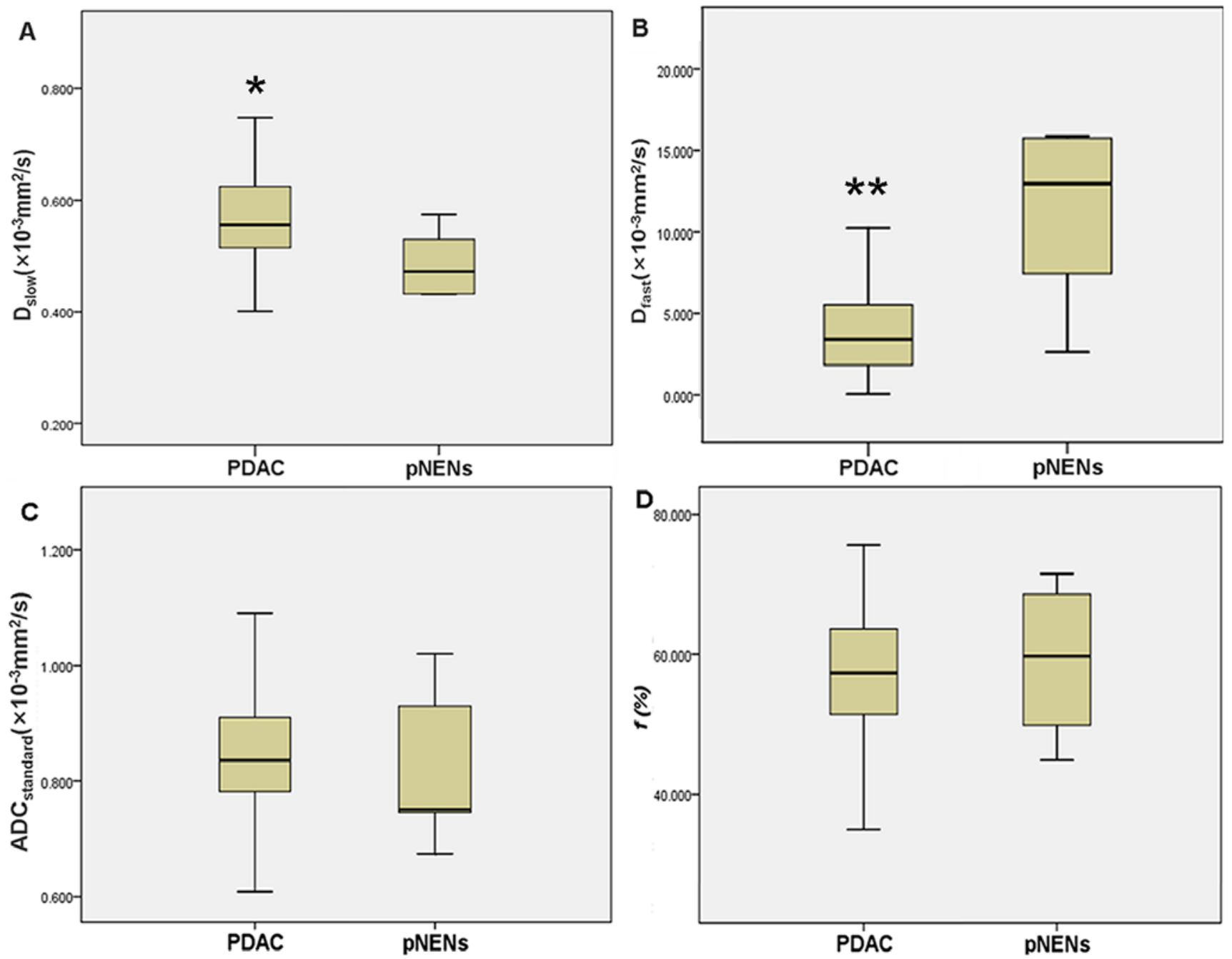

Figure 5. $\mathrm{ADC}_{\text {standard }}, \mathrm{D}_{\text {slow }}, \mathrm{D}_{\text {fast }}$ and $f$ values in pNENs and PDAC. The line represents the median. The central box represents the measurements from the lower (the 25 th percentile) to the upper (the 75 th percentile) quartile. Whiskers indicate the range from the minimum to the maximum parameter measurements. (A) * in the box-and-whisker plot revealed that the median $\mathrm{D}_{\text {slow }}$ value of PDAC was significantly higher compared with that of pNENs. (B) ${ }^{* *}$ in the box-and-whisker plot demonstrated that the median $\mathrm{D}_{\text {fast }}$ value of PDAC is significantly lower compared with that of pNENs. Box-and-whisker plots revealed that no significant differences were observed between PDAC and pNENs for (C) $\mathrm{ADC}_{\text {standard }}$ and (D) $f$. pNENs, pancreatic neuroendocrine neoplasms; PDAC, pancreatic ductal adenocarcinoma; $A D C$, apparent diffusion coefficient; $D_{\text {slow }}$, slow apparent diffusion coefficient; $D_{\text {fast }}$, fast apparent diffusion coefficient; $f$, fraction of fast apparent diffusion coefficient.

significantly higher compared with that of pNENs. This may be due to Kang et al using only nine b-values in IVIM-DWI; however, $15 \mathrm{~b}$-values were used in the present study. Therefore, in order to obtain high-quality IVIM-DWI, at least $10 \mathrm{~b}$-values should be used (40).
The present study demonstrated that the optimal AUC was achieved by combining $\mathrm{D}_{\text {slow }}$ and $\mathrm{D}_{\text {fast }}$ (AUC, 0.885), which was followed by $\mathrm{D}_{\text {fast }}$ and $\mathrm{D}_{\text {slow }}$ alone (AUC, 0.863 and 0.793 respectively). The highest specificity was identified for $\mathrm{D}_{\text {slow }}$ $(97.3 \%)$, which was closely followed by $\mathrm{D}_{\text {fast }}(91.9 \%)$. When 
Table V. Results of the receiver operating characteristic analysis for $\mathrm{D}_{\text {slow }}$ and $\mathrm{D}_{\text {fast }}$.

\begin{tabular}{lccccr}
\hline Coefficient & AUC (95\% CI) & Optimal cut-off & Sensitivity (\%) & Specificity (\%) & P-value \\
\hline $\mathrm{D}_{\text {slow }}$ & $0.793(0.655-0.895)$ & $\leq 0.472 \times 10^{-3} \mathrm{~mm}^{2} / \mathrm{sec}$ & 53.9 & 97.3 & $0.499^{\mathrm{a}}$ \\
$\mathrm{D}_{\text {fast }}$ & $0.863(0.736-0.944)$ & $>9.58 \times 10^{-3} \mathrm{~mm}^{2} / \mathrm{sec}$ & 69.2 & 91.9 & $0.757^{\mathrm{b}}$ \\
Combined $\mathrm{D}_{\text {slow }}$ and $\mathrm{D}_{\text {fast }}$ & $0.885(0.763-0.958)$ & - & 100 & 76.9 & $0.257^{\mathrm{c}}$ \\
\hline
\end{tabular}

${ }^{a} D_{\text {slow }}$ Vs. $D_{\text {fast }} ;{ }^{b} D_{\text {fast }}$ vs. Combined $D_{\text {slow }}$ and $D_{\text {fast }} ;{ }^{c} D_{\text {slow }}$ vs. Combined $D_{\text {slow }}$ and $D_{\text {fast }}$. AUC, area under the curve; CI, confidence interval; $D_{\text {slow }}$, slow apparent diffusion coefficient; $\mathrm{D}_{\text {fast }}$, fast apparent diffusion coefficient.

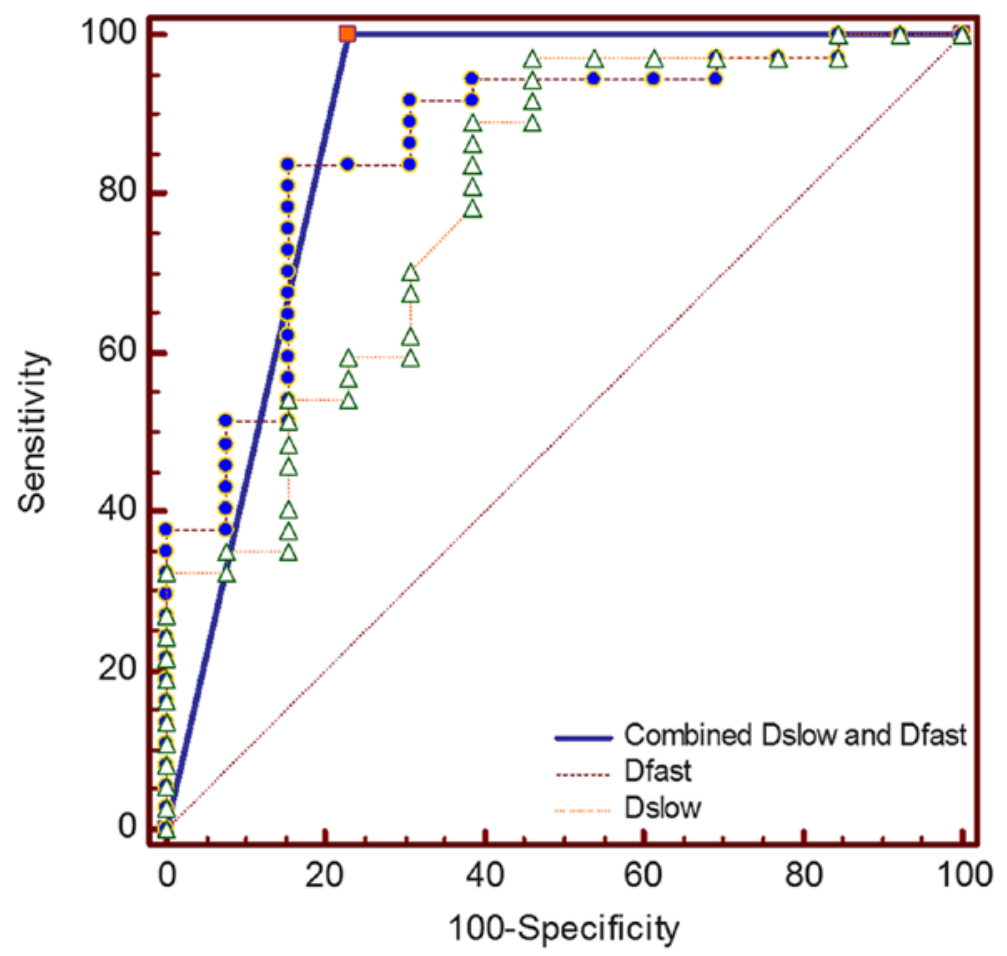

Figure 6. ROC curves of $\mathrm{D}_{\text {slow }}$ and $\mathrm{D}_{\text {fast }}$ for differentiating pNENs from PDAC. The diagonal line is the reference, which indicates the results for a test with 50\% sensitivity and $50 \%$ specificity. The highest AUC was obtained for combined $D_{\text {slow }}$ and $D_{\text {fast }}$, which was closely followed by $D_{\text {fast }}$ and $D_{\text {slow }}$ alone. ROC, receiver operating characteristics; $\mathrm{D}_{\text {slow }}$, slow apparent diffusion coefficient; $\mathrm{D}_{\text {fast }}$, fast apparent diffusion coefficient; AUC, area under the curve; pNENs, pancreatic neuroendocrine neoplasms; PDAC, pancreatic ductal adenocarcinoma.

$\mathrm{D}_{\text {slow }}$ and $\mathrm{D}_{\text {fast }}$ were combined, the sensitivity for differentiating high-grade pNENs from PDAC was $100.0 \%$. Therefore, $\mathrm{D}_{\text {slow }}$ and $\mathrm{D}_{\text {fast }}$ may be the ideal screening indicators in differentiating high-grade pNENs from PDAC.

There were certain limitations to the present study. For example, the sample size of the present study was relatively small, particularly regarding the limited number of high-grade pNENs due to its low incidence. However, all patients underwent surgery and were confirmed by histopathology. Secondly, the present study did not analyze the association between histopathology and IVIM-DWI parameters. This was due to the small sample size of different pathological grades pNENs and PDAC. Additional subjects need to be recruited in order to investigate the association between histopathology and IVIM-DWI parameters of pNENs and PDAC in further studies. In addition, dynamic contrast enhancement (DCE)-MRI was not performed in the present study due to the patients' preferences. The association between DCE-MRI and IVIM-DWI parameters will be investigated in our future studies. An additional limitation of the present study was that the b-values selected may have been suboptimal. Therefore, optimal b-values should be selected in any future studies to balance the least sampling time and the reliability of parameter estimation. Finally, a control group with normal pancreas was not included in the present study. This will be evaluated in further studies with larger populations.

In conclusion, the results of the present study demonstrated that the diffusion parameter $\mathrm{D}_{\text {slow }}$ and the perfusion parameter $\mathrm{D}_{\text {fast }}$ derived from IVIM-DWI may be reliable in differentiating high-grade pNENs from PDAC with high diagnostic accuracy. In addition, IVIM-DWI may be a potential technique to function as a surrogate biomarker in distinguishing pancreatic neoplasms. 


\section{Acknowledgements}

Not applicable.

\section{Funding}

The present study received funding from the National Natural Science Foundation of China (grant nos. NSFC 81220108011 and NSFC 81370039) and Shaanxi Social Development Science and Technology Plan (grant no. 2016SF-295).

\section{Availability of data and materials}

The datasets used and/or analyzed during the present study are available from the corresponding author upon reasonable request.

\section{Authors' contributions}

WM, MW and NL participated in the study conception and design. ZH, YT, QP and GZ collected and analyzed the patients' clinical data. WM, MW and JR collected and analyzed the patients' imaging data. WM, MW, ZH, YT, QP, GZ, JR, YH and NL participated in the interpretation of the patients' imaging data. WM and MW prepared the first draft of the manuscript. YH and NL revised the manuscript critically for important intellectual content. NL gave final approval of the version to be published. All authors revised and approved the final manuscript.

\section{Ethics approval and consent to participate}

Ethical approval was acquired from the Institutional Ethics Committee Board of Xijing Hospital (Xi'an, China), and written informed consent was obtained from all participants prior to collecting the data.

\section{Patient consent for publication}

Not applicable.

\section{Competing interests}

The authors declare that they have no competing interests.

\section{References}

1. Klimstra DS, Pitman MB and Hruban RN: An algorithmic approach to the diagnosis of pancreatic neoplasms. Arch Pathol Lab Med 133: 454-464, 2009.

2. Keutgen XM, Nilubol N and Kebebew E: Malignant-functioning neuroendocrine tumors of the pancreas: A survival analysis. Surgery 159: 1382-1389, 2016.

3. Kim JY, Hong SM and Ro JY: Recent updates on grading and classification of neuroendocrine tumors. Ann Diagn Pathol 29: 11-16, 2017.

4. Parekh JR, Wang SC, Bergsland EK, Venook AP, Warren RS, Kim GE and Nakakura EK: Lymph node sampling rates and predictors of nodal metastasis in pancreatic neuroendocrine tumor resections: The UCSF experience with 149 patients. Pancreas 41: 840-844, 2012.

5. Cloyd JM and Poultsides GA: Non-functional neuroendocrine tumors of the pancreas: Advances in diagnosis and management. World J Gastroenterol 21: 9512-9525, 2015

6. Kloppel G and Heitz PU: Pancreatic endocrine tumors. Pathol Res Pract 183: 155-168, 1988.
7. Philips S, Shah SN, Vikram R, Verma S, Shanbhogue AK and Prasad SR: Pancreatic endocrine neoplasms: A current update on genetics and imaging. Br J Radiol 85: 682-696, 2012.

8. Tesfaye AA, Kamgar M, Azmi A and Philip PA: The evolution into personalized therapies in pancreatic ductal adenocarcinoma: Challenges and opportunities. Expert Rev Anticancer Ther 18: 131-148, 2018.

9. Kourie H, Auclin E, Cunha AS, Gaujoux S, Bruzzi M, Sauvanet A, Lourenco N, Trouilloud I, Louafi S, El-Hajjar A, et al: Characteristic and outcomes of patients with pathologic complete response after preoperative treatment in borderline and locally advanced pancreatic adenocarcinoma: An AGEO multicentric retrospective cohort. Clin Res Hepatol Gastroenterol: Apr 24, 2019 doi: 10.1016/j.clinre.2019.03.007 (Epub ahead of print).

10. Couvelard A, O'Toole D, Turley H, Leek R, Sauvanet A, Degott C, Ruszniewski P, Belghiti J, Harris AL, Gatter K and Pezzella F: Microvascular density and hypoxia-inducible factor pathway in pancreatic endocrine tumours: Negative correlation of microvascular density and VEGF expression with tumour progression. Br J Cancer 92: 94-101, 2005.

11. d'Assignies G, Couvelard A, Bahrami S, Vullierme MP, Hammel P, Hentic O, Sauvanet A, Bedossa P, Ruszniewski P and Vilgrain V: Pancreatic endocrine tumors: \$umor blood flow assessed with perfusion CT reflects angiogenesis and correlates with prognostic factors. Radiology 250: 407-416, 2009.

12. Jang KM, Kim SH, Lee SJ and Choi D: The value of gadoxetic acid enhanced and diffusion-weighted MRI for prediction of grading of pancreatic neuroendocrine tumors. Acta Radiol 55: 140-148, 2014.

13. Herwick S, Miller FH and Keppke AL: MRI of islet cell tumors of the pancreas. AJR Am J Roentgenol 187: W472-W480, 2006.

14. Worhunsky DJ, Krampitz GW, Poullos PD, Visser BC, Kunz PL, Fisher GA, Norton JA and Poultsides GA: Pancreatic neuroendocrine tumours: Hypoenhancement on arterial phase computed tomography predicts biological aggressiveness. HPB (Oxford) 16: 304-311, 2014.

15. Rodallec M, Vilgrain V, Couvelard A, Rufat P, O'Toole D, Barrau V, Sauvanet A, Ruszniewski P and Menu Y: Endocrine pancreatic tumours and helical CT: Contrast enhancement is correlated with microvascular density, histoprognostic factors and survival. Pancreatology 6: 77-85, 2006.

16. Pasaoglu E, Dursun N, Ozyalvacli G, Hacihasanoglu E, Behzatoglu K and Calay O: Comparison of World Health Organization 2000/2004 and World Health Organization 2010 classifications for gastrointestinal and pancreatic neuroendocrine tumors. Ann Diagn Pathol 19: 81-87, 2015.

17. Karakus E, Helvacı A, Ekinci O and Dursun A: Comparison of WHO 2000 and WHO 2010 classifications of gastroenteropancreatic neuroendocrine tumors. Turk J Gastroenterol 25: 81-87, 2014.

18. Le Bihan D, Breton E, Lallemand D, Grenier P, Cabanis E and Laval-Jeantet M: MR imaging of intravoxel incoherent motions: Application to diffusion and perfusion in neurologic disorders. Radiology 161: 401-407, 1986.

19. Le Bihan D, Breton E, Lallemand D, Aubin ML, Vignaud J and Laval-Jeantet M: Separation of diffusion and perfusion in intravoxel incoherent motion MR imaging. Radiology 168: 497-505, 1988.

20. Le Bihan D and Turner R: The capillary network: A link between IVIM and classical perfusion. Magn Reson Med 27: 171-178, 1992.

21. Agarwal R, Brunelli SM, WilliamsK, Mitchell MD, Feldman HI and Umscheid CA: Gadolinium-based contrast agents and nephrogenic systemic fibrosis: A systematic review and meta-analysis. Nephrol Dial Transplant 24: 856-63, 2009.

22. Le Bihan D: Intravoxel incoherent motion perfusion MR imaging: A wake-up call. Radiology 249: 748-752, 2008.

23. Lee HJ, Rha SY, Chung YE, Shim HS, Kim YJ, Hur J, Hong YJ and Choi BW: Tumor perfusion-related parameter of diffusion-weighted magnetic resonance imaging: Correlation with histological microvessel density. Magn Reson Med 71: 1554-1558, 2014.

24. Kang KM, Lee JM, Yoon JH, Kiefer B, Han JK and Choi BI: Intravoxel incoherent motion diffusion-weighted MR imaging for characterization of focal pancreatic lesions. Radiology 270: 444-453, 2014.

25. Hwang EJ, Lee JM, Yoon JH, Kim JH, Han JK, Choi BI, Lee KB, Jang JY, Kim SW, Nickel MD and Kiefer B: Intravoxel incoherent motion diffusion-weighted imaging of pancreatic neuroendocrine tumors: Prediction of the histologic grade using pure diffusion coefficient and tumor size. Invest Radiol 49: 396-402, 2014 
26. Klau M, Mayer P, Bergmann F, Maier-Hein K, Hase J, Hackert T, Kauczor HU, Grenacher L and Stieltjes B: Correlation of histological vessel characteristics and diffusion-weighted imaging intravoxel incoherent motion-derived parameters in pancreatic ductal adenocarcinomas and pancreatic neuroendocrine tumors. Invest Radiol 50: 792-797, 2015.

27. Li ZS and Li Q: The latest 2010 WHO classification of tumors of digestive system. Zhonghua Bing Li Xue Za Zhi 40: 351-354, 2011 (In Chinese).

28. Thoeny HC and Ross BD: Predicting and monitoring cancer treatment response with diffusion-weighted MRI. J Magn Reson Imaging 32: 2-16, 2010.

29. Schnapauff D, Zeile M, Niederhagen MB, Fleige B, Tunn PU, Hamm B and Dudeck O: Diffusion-weighted echo-planar magnetic resonance imaging for the assessment of tumor cellularity in patients with soft-tissue sarcomas. J Magn Reson Imaging 29: 1355-1359, 2009

30. Muraoka N, Uematsu H, Kimura H, Imamura Y, Fujiwara Y, Murakami M, Yamaguchi A and Itoh H: Apparent diffusion coefficient in pancreatic cancer: Characterization and histopathological correlations. J Magn Reson Imaging 27: 1302-1308, 2008.

31. Wang Y, Chen ZE, Yaghmai V, Nikolaidis P, McCarthy RJ, Merrick L and Miller FH: Diffusion-weighted MR imaging in pancreatic endocrine tumors correlated with histopathologic characteristics. J Magn Reson Imaging 33: 1071-1079, 2011.

32. Vargas HA, Akin O, Franiel T, Mazaheri Y, Zheng J, Moskowitz C, Udo K, Eastham J and Hricak H: Diffusion-weighted endorectal MR imaging at $3 \mathrm{~T}$ for prostate cancer: Tumor detection and assessment of aggressiveness. Radiology 259: 775-784, 2011

33. Chandarana H, Lee VS, Hecht E, Taouli B and Sigmund EE: Comparison of biexponential and monoexponential model of diffusion weighted imaging in evaluation of renal lesions: Preliminary experience. Invest Radiol 46: 285-291, 2011.
34. Iima $M$ and Le Bihan D: Clinical intravoxel incoherent motion and diffusion MR Imaging: Past, Present, and Future. Radiology 278: 13-32, 2016

35. Jaster R and Emmrich J: Crucial role of fibrogenesis in pancreatic diseases. Best Pract Res Clin Gastroenterol 22: 17-29, 2008.

36. Padhani AR, Liu G, Koh DM, Chenevert TL, Thoeny HC, Takahara T, Dzik-Jurasz A, Ross BD, Van Cauteren M, Collins D, et al: Diffusion-weighted magnetic resonance imaging as a cancer biomarker: Consensus and recommendations. Neoplasia 11: 102-125, 2009.

37. Ginat DT, Mangla R, Yeaney G, Johnson M and Ekholm S: Diffusion-weighted imaging for differentiating benign from malignant skull lesions and correlation with cell density. AJR Am J Roentgenol 198: W597-W601, 2012.

38. Xu J, Liang Z, Hao S, Zhu L, Ashish M, Jin C, Fu D and Ni Q: Pancreatic adenocarcinomas: Dynamic 64-slice helical CT with perfusion imaging. Abdom Imaging 34: 759-766, 2009.

39. Takahashi Y, Akishima-Fukasawa Y, Kobayashi N, Sano T, Kosuge T, Nimura Y, Kanai Y and Hiraoka N: Prognostic value of tumor architecture, tumor-associated vascular characteristics, and expression of angiogenic molecules in pancreatic endocrine tumors. Clin Cancer Res 13: 187-196, 2007.

40. Lemke A, Stieltjes B, Schad LR and Laun FB: Toward an optimal distribution of $b$ values for intravoxel incoherent motion imaging. Magn Reson Imaging 29: 766-776, 2011.

This work is licensed under a Creative Commons Attribution-NonCommercial-NoDerivatives 4.0 International (CC BY-NC-ND 4.0) License. 